\title{
Origin of reactive oxygen species in human semen: spermatozoa or leucocytes?*
}

\author{
E. Kessopoulou ${ }^{1}$, M. J. Tomlinson ${ }^{2}$, C. L. R. Barratt ${ }^{1}$, A. E. Bolton ${ }^{2}$ \\ and I. D. Cooke ${ }^{1}$ \\ 'University Department of Obstetrics and Gynaecology, Jessop Hospital for Women, \\ Sheffield S3 7RE UK; and '2Division of Biomedical Sciences, Sheffield City Polytechnic, \\ Pond Street, Sheffield SI IWB, UK
}

\begin{abstract}
Summary. Peroxidative damage induced by reactive oxygen species (ROS) has been proposed as one of the major causes of defective sperm function. In previous studies of the production of ROS in semen, the contribution of contaminating leucocytes was not assessed. We determined the levels of ROS in 60 semen samples from men attending our infertility clinic and demonstrated by performing extraction experiments with antibody-coated magnetic beads that, within this unselected population of patients, leucocytes were the major source of ROS in the low-density Percoll fraction. Of the sperm motion parameters examined using computerized semen analysis, beat-cross frequency was the only one significantly affected by the ROS in semen.
\end{abstract}

Keiwords: antibody-coated magnetic beads; computerized sperm motility analysis; leucocytes; reactive oxygen species; spermatozoa; human

\section{Introduction}

High production of reactive oxygen species (ROS) in some ejaculates has been associated with defective sperm function (Aitken \& Clarkson, 1987; Aitken et al., 1989b) as it causes peroxidation of the polyunsaturated fatty acid content of the sperm plasma membrane (Jones et al., 1979). The result of this biochemical defect is that sperm cannot undergo the acrosome reaction and fuse with the oocyte (Aitken et al., 1989a), possibly owing to a loss of membrane fluidity (Ohyashiki et al., 1988). There are two potential sources of ROS in semen: spermatozoa and leucocytes (macrophages and neutrophils). Although the mechanism of ROS production by sperm has been extensively studied, the contribution of leucocytes to the seminal ROS has not been fully assessed. This is important because leucocytes, especially macrophages and neutrophils, are always present in semen (Barratt et al., 1990a).

The main function of these leucocyte populations is phagocytosis (Smith et al., 1990), a process associated with the production of ROS (Babior, 1978). From previous studies it appears that the sperm and leucocyte ROS-producing mechanisms involve similar pathways, i.e. a dependence on NADPH generated through the hexose monophosphate shunt (Aitken \& Ford, 1988), stimulation by phorbol esters and calcium ionophore (Aitken \& Clarkson, 1987). As pointed out by the first study that considered the potential of leucocytes to produce ROS (Aitken \& West, 1990), it is difficult to ascertain the relative contribution of spermatozoa and leucocytes to the ROS detected in semen. This could be achieved only if 'leucocyte extraction experiments' are performed from sperm preparations in which the concentrations of ROS are measured before and after the removal of the leucocyte population (Barratt et al., 1990a; Aitken \& West, 1990).

We used antibody-coated magnetic beads to extract leucocytes from semen samples fractionated on discontinuous Percoll gradients to determine the source of reactive oxygen species in semen. In

\footnotetext{
*Part of this work was presented at a meeting of the Society of the Study of Fertility (Kessopoulou et al., 1990).
} 
the course of the experiments a simple chemiluminescent assay was used to follow the production of reactive oxygen species in the fractions that were generated from a double-step Percoll gradient (Aitken et al., 1989a). The chemiluminescent signals were recorded in the leucocyte-rich fraction before and after the extraction of the leucocytes with immunomagnetic beads. Monoclonal antibody (mAb) staining was used to detect seminal leucocytes (Tomlinson et al., 1990). Quantitative motility analysis was used to evaluate any effect of ROS on sperm function, as this technique has been shown to indicate the fertilizing capacity of human sperm in a donor insemination (DI) programme (Irvine \& Aitken, 1986; Holt et al., 1989).

\section{Materials and Methods}

\section{Experimental design}

Semen samples were liquefied and then subjected to manual and computerized semen analysis. Double-step discontinuous Percoll gradients ( 40 and $80 \%$ ) were used to separate the semen samples and ROS were determined with a simple chemiluminescent assay. Contaminating leucocytes were extracted from the low-density layers of the Percoll gradients with magnetic beads coated with a common leucocyte antibody. The chemiluminescent response of the isolated leucocytes and the 'sperm fraction' was recorded to determine the source of the radicals. Leucocytes were identified in whole semen and in the Percoll-generated fractions with $\mathrm{mAbs}$ and the alkaline phosphatase anti-alkaline phosphatase (APAAP) staining method.

\section{Semen analysis}

Semen samples were obtained from 60 patients attending the University Research Clinic, Jessop Hospital for Women, Sheffield, UK. The samples were liquefied at $37^{\circ} \mathrm{C}$ for $30 \mathrm{~min}$, the volume and $\mathrm{pH}$ of the samples were then measured and smears were prepared for morphological analysis (Barratt et al., 1988). In addition, the samples were analysed for quantitative motility using a Hamilton Thorn Motility Analyzer (Hamilton Thorn Research Inc., Danvers, USA). A $10 \mu \mathrm{l}$ aliquant of semen was loaded into a $20 \mu \mathrm{m}$ Hamilton Thorn microslide on a heated stage $\left(37^{\circ} \mathrm{C}\right)$ and was examined under an Olympus BH-2 microscope (Olympus Optical Co, Tokyo, Japan) using phase contrast optics $(20 \times$ objective). The analyser set-up is outlined in Table 1 . The performance of the analyser was continuously checked using the play-back function. A different set-up was used for samples with high round-cell contamination.

Table 1. Hamilton Thorn analyser set-up used to analyse whole semen samples and Percoll-generated fractions without round-cell contamination

\begin{tabular}{|c|c|}
\hline Frames at frame rate & $20 / 25 \mathrm{~s}^{1}$ \\
\hline Minimum contrast* & 6 \\
\hline Minimum size & $4 \mu \mathrm{m}^{2}$ \\
\hline Low/high size gates & $0.3 \mu \mathrm{m}^{2} \quad 1.7 \mu \mathrm{m}^{2}$ \\
\hline Low/high intensity gates* & $0.3 \quad 1 \cdot 5$ \\
\hline Head size of nonmotile spermatozoa & $6 \mu \mathrm{m}^{2}$ \\
\hline Head intensity of nonmotile spermatozoa* & 115 \\
\hline Medium path velocity $(V)$ & $25 \mu \mathrm{m} \mathrm{s}$ \\
\hline Low path velocity & $10 \mu \mathrm{m} \mathrm{s}^{1}$ \\
\hline Slow spermatozoa motile & No \\
\hline Threshold straightness & $80 \%$ \\
\hline
\end{tabular}

*Units of brightness above background intensity.

\section{Determination of reactive oxygen species}

The semen samples were fractionated on double-step discontinuous Percoll gradients prepared according to Aitken et al. (1989a) using Earle's balanced salts (Gibco Ltd, Middlesex, UK) supplemented with 0.3\% bovine serum 
albumin (Fraction V; Sigma. Dorset, UK) (Earle’s BSA) instead of BWW (Biggers et al., 1971). Each gradient consisted of $3 \mathrm{ml} 40 \%$ Percoll loaded on top of $3 \mathrm{ml} 80 \%$ Percoll (Sigma, Dorset, UK) in a $15 \mathrm{ml}$ conical sterile centrifuge tube. A sufficient volume of semen $(>1 \mathrm{ml})$ was layered on top of the gradient.

The gradients were centrifuged at $500 \mathrm{~g}$ for $20 \mathrm{~min}$ and the seminal plasma was then removed and two cellular layers from the $40-80 \%$ interphase and the $80 \%$ base were collected. These cellular layers were subsequently washed with Earle's BSA at $500 \mathrm{~g}$ for $5 \mathrm{~min}$ and resuspended with Earle's BSA to a concentration of $10 \times 10^{6}$ spermatoz.oa $\mathrm{ml}$ '. The cellular layers were also analysed using quantitative motility analysis.

Reactive oxygen species production was determined according to Aitken et al. (1987): $1 \mu \mathrm{l}$ of the chemiluminescent probe luminol ( $100 \mathrm{mmol} l^{\prime}$ in dimethylsulfoxide) was added to $400 \mu$ l spermatozoa suspensions at $10 \times 10^{6} \mathrm{ml} \mathrm{l}^{-1}$ and the response monitored on a 1251 BioOrbit luminometer $\left(37^{\circ} \mathrm{C}\right)$ (BioOrbit UK Ltd, Berkshire, UK) driven by an IBM-compatible personal computer.

Extraction experiments with antibody-coated magnetic beads were performed to clarify the source of the radicals. This particular assay was performed for only 25 of the 60 samples as the volume of the samples was limiting. A 5-20 $\mu 1$ suspension of magnetic beads (Dynal UK Ltd, Wirral, Merseyside, UK) coated with a common anti-leucocyte antibody (HLe-1, CD45, Becton Dickinson, Cowley, Oxford, UK) was added to $400 \mu \mathrm{l}$ of the $40-80 \%$ fraction to extract the contaminating leucocytes. After incubation for $1 \mathrm{~h}$ with continuous agitation at room temperature, the beads with the adherent cells were removed with a magnet (Magnetic Particle Concentrator MPC-1, Dynal UK Ltd, Wirral, Merseyside, UK) and were washed twice with Earle's BSA to remove any contaminating spermatozoa.

Determination of ROS of the leucocytes that were adherent to the beads and the nonadherent cells (spermatozoa and germinal cells) was then carried out as previously described.

\section{Identification and quantification of leucocytes}

Preparation of semen smears. Smears of the initial sample and of the Percoll-generated fractions were reacted with $\mathrm{mAbs}$ and visualized using the APAAP method to determine the numbers and subtypes of the leucocytes present.

Liquefied semen was mixed with phosphate-buffered saline (PBS, Gibco Ltd, Middlesex, UK) and centrifuged at $600 \mathrm{~g}$ for $10 \mathrm{~min}$ to remove the seminal plasma. This procedure was repeated twice and the cellular pellet was resuspended in PBS to the original volume of the semen sample. This suspension was diluted (1:5 and 1:2) in PBS and aliquots of $5 \mu \mathrm{l}$ transferred onto individual wells of a PTFE-coated multiwell slide (C. A. Hendley, Essex, UK).

The Percoll-generated fractions were washed with PBS three times and centrifuged at $600 \mathrm{~g} .5 \mu \mathrm{l}$ aliquants were spotted in duplicate onto multiwell slides. Slides were air-dried, wrapped in aluminium foil and stored at $-20^{\circ} \mathrm{C}$ before staining.

Positive and negative control slides were also prepared from human peripheral biood leucocytes that were isolated from whole heparinized blood using density gradient centrifugation (Histopaque 1119/1077, Sigma, Dorset, UK) and stored under identical conditions. For the negative controls, the primary antibodies were omitted from the staining regimen.

$\boldsymbol{m} \boldsymbol{A} \boldsymbol{b}$ staining. Slides were allowed to thaw for $30 \mathrm{~min}$ before fixing in acetone:methanol (1:1) for $90 \mathrm{~s}$ and washing in Tris-buffered saline (TBS, $0.05 \mathrm{~mol}^{-1}$ Tris, $0.15 \mathrm{~mol} \mathrm{NaCll}$ ', $\mathrm{pH} 7.6$ ) for $5 \mathrm{~min}$. All slides were incubated in normal rabbit serum ( $1: 5)$ for 20 min to saturate nonspecific binding sites. A panel of mAbs that have all been shown to work well on semen smears (Wolff et al., 1990) was used to detect leucocytes and their subsets in semen (Table 2).

Table 2. Monoclonal antibodies (mAb) used to identify leucocytes in human semen

\begin{tabular}{lll}
\hline MAb & \multicolumn{1}{c}{ Specificity } & \multicolumn{1}{c}{ Source } \\
\hline HLe-1 & All leucocytes & Becton Dickinson, Oxford, UK (BD) \\
Leu-2a & Helper/inducer T lymphocytes & (BD) \\
Leu-3a & Suppressor/cytotoxic T lymphocytes & (BD) \\
Macrophage & Monocytes-macrophages & Dako, High Wycombe, UK (D) \\
Pan B cell & B lymphocytes & (D) \\
FMC-10 & Polymorphonuclear leucocytes & Serotec, Oxford, UK \\
\hline
\end{tabular}

Slides were stained using the APAAP technique with a modification of the method described by Mason (1985) which was first used to identify round cells in semen by Schobel et al. (1989). Ten microlitres of primary antibody (at a predetermined dilution) was spread over each well and incubated for $30 \mathrm{~min}$ at room temperature.

After a $2 \mathrm{~min}$ wash with TBS, slides were incubated for $30 \mathrm{~min}$ with a rabbit anti-mouse secondary antibody (Dako, High Wycombe, UK) diluted 1:25. The slides were washed once more in TBS, and the smears finally developed with an APAAP complex diluted 1:40 for $1 \mathrm{~h}$ (Dako, High Wycombe, UK). Enhancement of the labelling reaction was then ensured by repeating the incubation with secondary antibody followed by the APAAP complex for 10 min each. 
Alkaline phosphatase activity was determined by incubation with a substrate containing $0.5 \mathrm{mmol}$ naphthol As-MX phosphate $\mathrm{ml}^{1}, 2 \%$ dimethyl-formamide, $0.01 \mathrm{mmol}$ levamisole l ' (for inhibition of endogenous alkaline phosphatase activity) and 3.9-mm Fast Red TR in Tris buffer ( $\mathrm{pH} \mathrm{8.2)} \mathrm{for} 20 \mathrm{~min}$. Slides were then counterstained with haematoxylin and mounted in Apathy's mounting medium (BDH, Poole, Dorset, UK).

Positive red-stained cells were counted on a Leitz Laborlux microscope (Leitz, Germany) under bright field illumination $(40 \times$ objective). The total number of positive cells on duplicate spots was recorded, averaged and multiplied by the dilution factor $(\times 200)$ to give the number of leucocytes per $\mathrm{ml}$ of semen (Cohen, 1971). Numbers of leucocytes in the Percoll fractions were expressed per $10 \times 10^{6}$ spermatozoa.

\section{Statistical analysis}

The data were normalized using logarithmic transformations and nonparametric tests (Mann-Whitney and Wilcoxon signed rank test) were performed. The relationship between different parameters was determined using linear regression analysis. All the tests were performed using the Statistical Package for the Social Sciences (SPSS) on an IBM-compatible personal computer.

\section{Results}

The semen characteristics obtained for the unfractionated samples and the Percoll-generated fractions are shown in Tables 3 and 4 respectively. Chemiluminescent determination of the basal ROS levels in the Percoll-generated fractions revealed a wide range of time-dependent activity with a significant difference $(P<0.0001)$ between the low $(40-80 \%)$ and high $(80 \%)$ density fractions (Fig. 1).

Table 3. Semen parameters of unfractionated samples examined for reactive oxygen species

\begin{tabular}{|c|c|c|}
\hline Semen parameter & Median & Range \\
\hline Volume (ml) & $3 \cdot 5$ & $1.09 \cdot 0$ \\
\hline $\mathrm{pH}$ & 7.9 & $7 \cdot 4-9 \cdot 0$ \\
\hline Density $\left(10^{6}\right.$ spermatozoa $\left.\mathrm{ml}{ }^{1}\right)$ & $44 \cdot 0$ & $2 \cdot 0 \quad 180$ \\
\hline \multicolumn{3}{|l|}{ Sperm motility (\%) } \\
\hline grade I $(\mathrm{V}>25)$ & $25 \cdot 0$ & $0-33$ \\
\hline grade II $(10<\mathrm{V}<25)$ & $9 \cdot 0$ & $0-62$ \\
\hline grade III $(0<\mathrm{V}<10)$ & $7 \cdot 0$ & 3-38 \\
\hline grade IV $(V=0)$ & $59 \cdot 0$ & $9-94$ \\
\hline Path velocity $\left(\mu \mathrm{ms}{ }^{\prime}\right)$ & $41 \cdot 0$ & $18-61$ \\
\hline Progressive velocity ( $\left.\mu \mathrm{m} \mathrm{s}^{\prime}\right)$ & 33.0 & $11-53$ \\
\hline Lateral head displacement $(\mu \mathrm{m})$ & $2 \cdot 7$ & $1-4$ \\
\hline Curvilinear velocity $(\mu \mathrm{ms})$ & $49 \cdot 0$ & 23* 73 \\
\hline Beat-cross frequency $(\mathrm{Hz})^{*}$ & $8 \cdot 2$ & $2-15$ \\
\hline Linearity $(\%)$ & $64 \cdot 0$ & $40-84$ \\
\hline Straightness $(\%)$ & $79 \cdot 0$ & $57-91$ \\
\hline \multicolumn{3}{|l|}{ Sperm morphology $(\%)$} \\
\hline Ideal forms & $41 \cdot 5$ & $2-76$ \\
\hline Head defects & $40 \cdot 0$ & $0-98$ \\
\hline Midpiece defects & $13 \cdot 5$ & $0-60$ \\
\hline Tail defects & $13 \cdot 5$ & 0.62 \\
\hline
\end{tabular}

The identification of seminal leucocytes with monoclonal antibodies and the APAAP staining method in combination with the fixed volume preparations were extremely reliable $(\mathrm{CV}<10 \%)$. A large variation in the number of leucocytes per $\mathrm{ml}$ of semen (median 13000, range 400-2 710 000) was detected with only two samples exhibiting $>1 \times 10^{6}$ leucocytes. The predominant leucocyte 
Table 4. Semen parameters of Percoll-generated fractions

\begin{tabular}{lcc}
\hline Semen parameter & $\begin{array}{c}40-80 \% \text { Percoll fraction } \\
\text { Median (Range) }\end{array}$ & $\begin{array}{c}80 \% \text { Percoll fraction } \\
\text { Median (Range) }\end{array}$ \\
\hline $\begin{array}{l}\text { Sperm motility }(\%) \\
\text { grade I }(\mathrm{V}>25)\end{array}$ & $\begin{array}{l}\text { ( }) \\
\text { grade II }(10<\mathrm{V}<25)\end{array}$ & $11 \cdot 0(3-83)$ \\
grade III $(0<\mathrm{V}<10)$ & $5 \cdot 0(0-26)$ & $5 \cdot 0(0-12)$ \\
grade IV $(\mathrm{V}=0)$ & $3 \cdot 0(0-85)$ & $41 \cdot 0(9-95)$ \\
Path velocity $\left(\mu \mathrm{m} \mathrm{s} \mathrm{s}^{\prime}\right)$ & $84 \cdot 0(12-98)$ & $44 \cdot 0(18-79)$ \\
Progressive velocity $\left(\mu \mathrm{m} \mathrm{s}^{\prime}\right)$ & $33 \cdot 0(20-68)$ & $36 \cdot 0(9-72)$ \\
Lateral head displacement $(\mu \mathrm{m})$ & $26 \cdot 0(14-98)$ & $3 \cdot 6(2-6)$ \\
Curvilinear velocity $\left(\mu \mathrm{m} \mathrm{s}^{-1}\right)$ & $3 \cdot 1(1-6)$ & $60 \cdot 0(26-98)$ \\
Beat-cross frequency $(\mathrm{Hz})$ & $44 \cdot 0(26-79)$ & $10 \cdot 3(4-15)$ \\
Linearity $(\%)$ & $9 \cdot 3(2-25)$ & $60 \cdot 0(35-83)$ \\
Straightness $(\%)$ & $57 \cdot 0(16-87)$ & $76 \cdot 0(51-88)$ \\
\hline
\end{tabular}

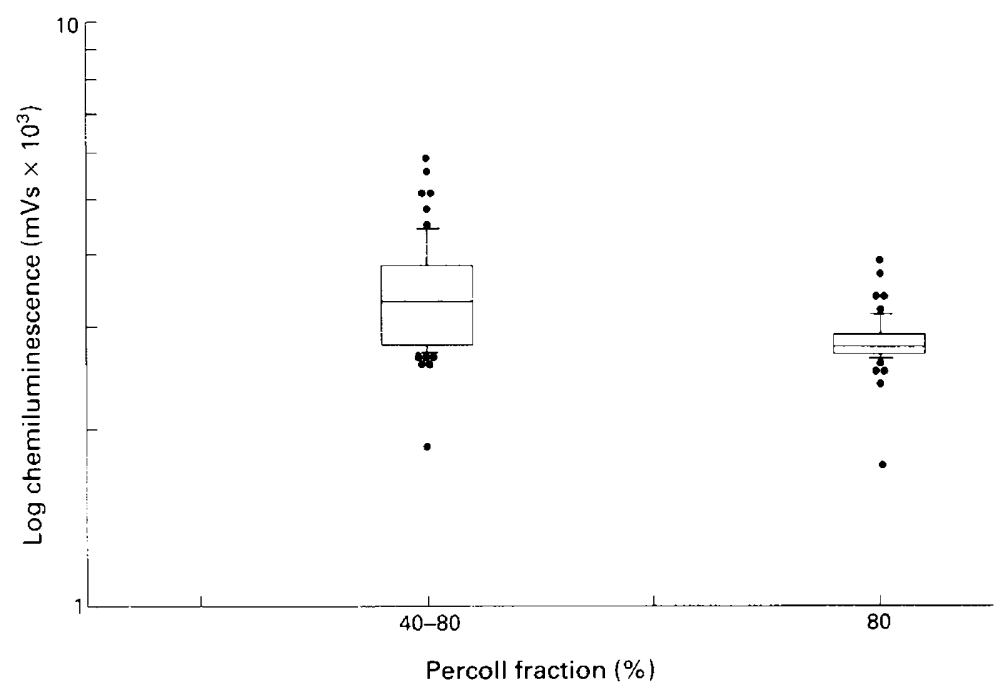

Fig. 1. Comparison of production of reactive oxygen species in the $40-80 \%$ and $80 \%$ Percoll fractions of human semen over $10 \mathrm{~min}$. ROS expressed as chemiluminescent signal. Boxes indicate the 25 and 75 percentiles while the horizontal line within the box indicates the 50 percentile. Vertical lines indicate 10 and 90 percentile and dots indicate values outside this range. ROS of the two Percoll fractions are significantly different $(P<0.0001)$.

type, the neutrophil, was detected in almost every sample and accounted for $81 \%$ of the total leucocyte counts. Cells of the monocyte-macrophage series accounted for $16 \%$ of the total leucocyte numbers whereas $\mathrm{T}$ and $\mathrm{B}$ lymphocytes were rarely detected and then only in small numbers.

In terms of leucocyte concentrations there was a highly significant difference $(P<0.0001)$ between the low and high density Percoll fractions. The median leucocyte number per $10 \times 10^{6}$ spermatozoa in the $40-80 \%$ Percoll fraction was 4221 (range $0-326333$ ) while in the $80 \%$ fraction it was 143 (range $0-55000$ ).

Extraction experiments with magnetic antibody-coated beads were performed from the leucocyte-rich Percoll fraction $(40 / 80 \%$ ) for 25 out of the 60 samples as the volume of the sample was limiting. The integrated response in the ROS assay from the leucocyte fraction for a $10 \mathrm{~min}$ 


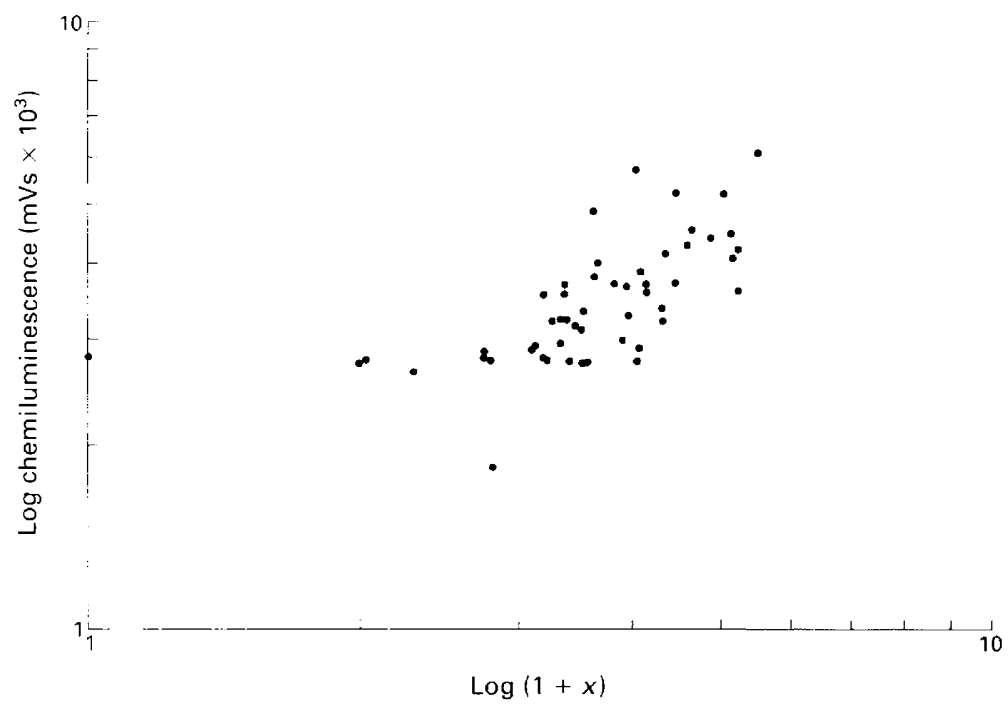

Fig. 2. Log-log plot of reactive oxygen species over $10 \mathrm{~min}$ in $40-80 \%$ Percoll fraction and numbers of leucocytes $(r=0 \cdot 65, P<0 \cdot 001)$. $x$ : number of leucocytes $\times 10^{3}$ per $10^{7}$ spermatozoa.

period was higher $(P<0.001)$ than that from the 'sperm' fraction and accounted for $65 \%$ (median and range $50-76 \%$ ) of the overall response. There was negligible sperm contamination of the leucocyte fraction. The average recovery of leucocytes from the $40-80 \%$ Percoll fraction with the beads was $88 \%$ (range $75-97 \%$ ).

Linear regression analysis, which was performed after appropriate logarithmic transformations $(\log x, \log (1+x))$ of both the chemiluminescent response and the leucocyte numbers in the $40-80 \%$ Percoll fractions, revealed that there was a strong correlation between ROS levels and leucocyte numbers $(r=0.65, P<0.001)$ (Fig. 2).

Of the motility parameters analysed only beat-cross frequency was significantly negatively correlated with ROS levels $(r=-0.48, P<0 \cdot 001)$ (Table 3$)$. There was no significant correlation between sperm morphology and ROS (Table 3 ).

There was no significant difference in any of the parameters or the ROS between oligozoospermic semen $\left(<20 \times 10^{6}\right.$ sperm $\left.\mathrm{ml}^{-1}\right)$, which comprised the semen of $15 \%$ of the population of patients, and normal-density semen.

\section{Discussion}

In this study we examined the contribution of seminal leucocytes to the levels of reactive oxygen species detected in semen using a novel method to extract leucocytes from sperm preparations. We provided clear evidence that, in an unselected population of patients attending an infertility clinic for the first semen sample, leucocytes are the main source of ROS in the low-density Percoll fraction leaving spermatozoa to account for a lower proportion.

The methods used to identify and quantify seminal leucocytes were reproducible. Furthermore the application of magnetic antibody-coated beads enabled us to isolate quite effectively most of the leucocytes from sperm preparations (recovery $>75 \%$ ).

In all previous studies that have examined the production of ROS in whole semen, density gradient generated fractions, or swim-up preparations, the possibility of a leucocyte origin was excluded either on the basis of $<1 \times 10^{6} \mathrm{ml}{ }^{1}$ leucocyte contamination or less than $1 \%$ contamination (Aitken $\&$ Clarkson, 1987; Alvarez et al., 1987; D’Agata et al., 1990). However, these authors did not provide 
sufficient detail of the methods used to identify and quantify leucocytes. Identification of seminal leucocytes with standard cytochemical techniques such as the peroxidase method has been proved inadequate and inaccurate as it identifies only leucocytes with an intact peroxidase enzyme (mainly neutrophils) (Barratt et al., 1990b).

The data we obtained using an accurate and reliable method to identify seminal leucocytes demonstrate that a leucocyte contamination of a semen sample of less than $1 \times 10^{6} \mathrm{ml}^{-1}$ or even less than $1 \%$ (median contamination in $40-80 \%$ Percoll fraction $0.04 \%$, range $0-3.26 \%$ ) can be effective in producing ROS. Our observations are in accordance with previous in vitro experiments that showed that leucocytes are more copious producers of ROS than are sperm as they can produce at least 100 times more ROS than spermatozoa (Ford, 1990).

We observed little negative correlation between ROS and motility parameters. This poor correlation might not be surprising as in a previous study Aitken et al. (1989a) showed that, although low levels of induced peroxidation prevented sperm penetration of zona-free hamster eggs, sperm motility was not affected. It therefore appears that motility analysis is not an adequate predictor of sperm function (Tomlinson et al., 1990) and should be performed in conjunction with more sophisticated tests such as zona-binding and induction of acrosome reaction.

Our findings that leucocytes are the main source of ROS in semen are important in view of the well-documented antioxidant effects of the seminal plasma. The synergistic action of ROSscavenging mechanisms in seminal plasma can protect spermatozoa when ROS production is not excessive (Jones et al., 1979; Alvarez \& Storey, 1989; Jeulin et al., 1989; Bouvet et al., 1990). Furthermore ROS have a very short half life and they would be rapidly degraded (Lunec, 1989).

The effectiveness of these 'extraspermatozoal' ROS on sperm function might depend on the time of contact of leucocytes with spermatozoa which makes the issue of their origin important and the need for human studies imperative (Barratt et al., 1990a).

Nevertheless in cases where the leucocyte-derived ROS affect the functional ability of the spermatozoa either because the seminal plasma protective mechanisms are overwhelmed or depleted, in vitro or in vivo therapy with antioxidants should be considered (Aitken, 1989).

We suggest that careful identification and evaluation of leucocyte numbers and determination of spermatozoal and seminal plasma protective mechanisms should be performed in future experiments that investigate the effect of ROS on sperm function.

The authors wish to acknowledge R. J. Aitken and W. C. L. Ford for their constructive criticism. The excellent technical support of A. White and C. F. Dalton is greatly appreciated. M. J. Tomlinson has a grant from the Medical Research Council. The study was supported by Infertility Research Trust and Trent Regional Health Authority.

\section{References}

Aitken, R.J. (1989) Editorial: The role of free oxygen radicals and sperm function. Int. J. Androl. 12, 95-97.

Aitken, R.J. \& Clarkson, J.S. (1987) Cellular basis of defective sperm function and its association with the genesis of reactive oxygen species by human spermatozoa. J. Reprod. Fert. 81, 459-469.

Aitken, R.J. \& Ford, W.C.L. (1988) Investigation of ccllular mechanisms regulating the release of superoxide anion by human spermatozoa. J. Reprod. Fert. Ahst Ser. 1, 40.

Aitken, R.J. \& West, K.M. (1990) Analysis of the relationship between reactive oxygen species production and leucocyte infiltration in fractions of human semen separated on Percoll gradients. Int. J. Androl. 13, 433-45l
Aitken, R.J., Clarkson, J.S. \& Fishel, S. (1989a) Generation of reactive oxygen species, lipid peroxidation, and human sperm function. Biol. Reprod. 40, 183-197.

Aitken, R.J., Clarkson, J.S., Hargreave, T.B., Irvine, D.S. \& Wu, F.C.W. (1989b) Analysis of the relationship between defective sperm function and the generation of reactive oxygen species in cases of oligozoospermia. J. Androl. 10, 214-220.

Alvarez, J.G. \& Storey, B.T. (1989) Role of glutathione peroxidase in protecting mammalian spermatozoa from loss of motility caused by spontaneous lipid peroxidation. Gamete Res. 23, 77-90.

Alvarez, J.G., Touchstone, J.C., Blasco, L. \& Storey, B.T. (1987) Spontaneous lipid peroxidation and production of hydrogen peroxide and superoxide in 
human spermatozoa; superoxide dismutase as the major enzyme protectant against oxygen toxicity. $J$. Androl. 8, 338-348.

Babior, B.M. (1978) Oxygen dependent microbial killing by phagocytes. New Engl. J. Med. 298, 659-668.

Barratt, C.L.R., Dunphy, B.C., Thomas, E.J. \& Cooke, I.D. (1988) Semen characteristics of 49 fertile males. Andrologia 20, 264-269.

Barratt, C.L.R., Bolton, A.E. \& Cooke, I.D. (1990a) Functional significance of white blood cells in the male and female reproductive tract. Hum. Reprod. 5, 639-648.

Barratt, C.L.R., Robinson, A., Kinghorn, G.R., White, A., Harrison, P.E., Kessopoulou, E. \& Cooke, I.D. (1990b) Seminal peroxidase positive cells are not an adequate predictor of asymptomatic urethral infection. Int. J. Androl. 13, 361-368.

Biggers, J.D., Whitten, W.K. \& Whittingham, D.G. (1971) The culture of mouse embryos in vitro. In Methods in Mammalian Embryology, pp. 86-116. Ed. J.C. Daniel. Freeman, San Francisco.

Bouvet, J.P., D'Azambuja, S., Roux, C., Lebrun, L. \& Pillot, J. (1990) Human seminal plasma suppresses the chemiluminescence of polymorphonuclears without modifying phagocytosis. Am. J. Reprod. Immunol. 23, 64-68.

Cohen, J. (1971) The comparative physiology of gamete populations. In Advances in Comparative Physiology and Biochemistry, vol. 4, pp. 267-380. Ed. O. Lowenstein. Academic Press, New York.

D'Agata, R., Vicari, E., Moncada, M.L., Sidoti, G., Calogero, A.E., Fornito, M.C., Minicipalli, E., Mongioi, A. \& Polosa, P. (1990) Generation of reactive oxygen species in subgroups of infertile men. Int. J. Androl. 13, 344-351.

Ford, W.C.L. (1990) The role of oxygen free radicals in the pathology of human spermatozoa: implications of IVF. In Clinical IVF Forum: Current Views in Assisted Reproduction, pp. 123--139. Eds P. L. Matson \& B. A. Lieberman. Manchester University Press, UK.

Holt, W.V., Shenfield, F., Leonard, T., Hartman, T.D., North, R.D. \& Moore, H.D. (1989) The value of sperm swimming speed measurements in assessing the fertility of human frozen semen. Hum. Reprod. 4, 292-297.
Irvine, W.V. \& Aitken, R.J. (1986) Predictive value of invitro sperm function tests in the context of an AID service. Hum. Reprod. 1, 539-545.

Jeulin, C., Soufir, J.C., Weber, P., Laval-Martin, D. \& Calvayrae, R. (1989) Catalase activity in human sperm and seminal plasma. Gamete Res. 24, 185-196.

Jones, R., Mann, T. \& Sherins, R. (1979) Peroxidative breakdown of phospholipids in human spermatozoa, spermicidal properties of fatty acid peroxides and protective action of seminal plasma. Fert. Steril. 31, $531-537$.

Kessopoulou, E., Barratt, C.L.R. \& Cooke, I.D. (1990) Reactive oxygen species and seminal leucocytes. $J$. Reprod. Fert. Abst Ser. 6, Abst 1.

Lunec, J. (1989) Oxygen radicals: their measurement in vivo. Anal. Proceed. 26, 130-131.

Mason, D.Y. (1985) Immunocytochemical labelling of monoclonal antibodies by the APAAP immunoalkaline phosphatase technique. In Techniques in Immunocytochemistry 3, pp. 25-42. Eds G. R. Bullock \& P. Petrusz. Academic Press, London.

Ohyashiki, T., Ohtsuka, T. \& Mohri, T. (1988) Increase of the molecular rigidity of the protein conformation in the intestinal brush-border membranes by lipid peroxidation. Biochim. Biophys. Acta 13, 383-392.

Schobel, W.A., Schieferstein, G. \& Uchanska-Zíegler, B. (1989) Immunocytochemical characterisation of round cells in human semen using monoclonal antibodies and the APAAP-technique. Andrologia 21, 370-376.

Smith, D.C., Barratt, C.L.R. \& Williams, M.A. (1990) The characterization of non-sperm cells in the ejaculates of fertile men using transmission electron microscopy. Andrologia 21, 319-333.

Tomlinson, M.J., Barratt, C.L.R., Bolton, A.E. \& Cooke, I.D. (1990) Seminal leucocytes and semen quality. J. Reprod. Fert. Abst Ser. 6, Abst 61 .

Wolff, M.D., Politch, J.A., Martinez, A., Haimovici, F., Hill, J.A. \& Anderson, D.J. (1990) Leucocytospermia is associated with poor semen quality. Fert. Steril. 53, $528-536$. 